\title{
EVIDENCE OF FIELD TWISTING IN INTEGRATED PROFILES OBSERVED OVER A WIDE FREQUENCY RANGE
}

\author{
V. A. Izvekova, A. D. Kuz'min \\ Radio Astronomy Department, Lebedev Physical Institute \\ A. G. LYNE \\ Nuffield Radio Astronomy Laboratories, University of Manchester \\ YU. P. ShITOV \\ Radio Astronomy Department, Lebedev Physical Institute \\ F. Graham-Smith \\ Nuffield Radio Astronomy Laboratories, University of Manchester
}

\section{Introduction}

The evolution of integrated pulse profiles from high to low frequencies does not conform exactly to the simple model of a dipole field. Several groups (Davies et al. 1984, Kuz'min 1986, Shitov, Malofeev, and Izvekova 1988) have suggested large field twisting due to the rotation of the pulsar, which leads to a delay in pulse arrival times for low frequencies whose sources are located in the outer parts of the magnetosphere. We are, therefore, comparing profiles of about 30 pulsars, observed at frequencies from 40 to $1400 \mathrm{MHz}$. Our preliminary analysis shows that significant delay at low frequencies occurs in many pulsars and we present here some typical examples.

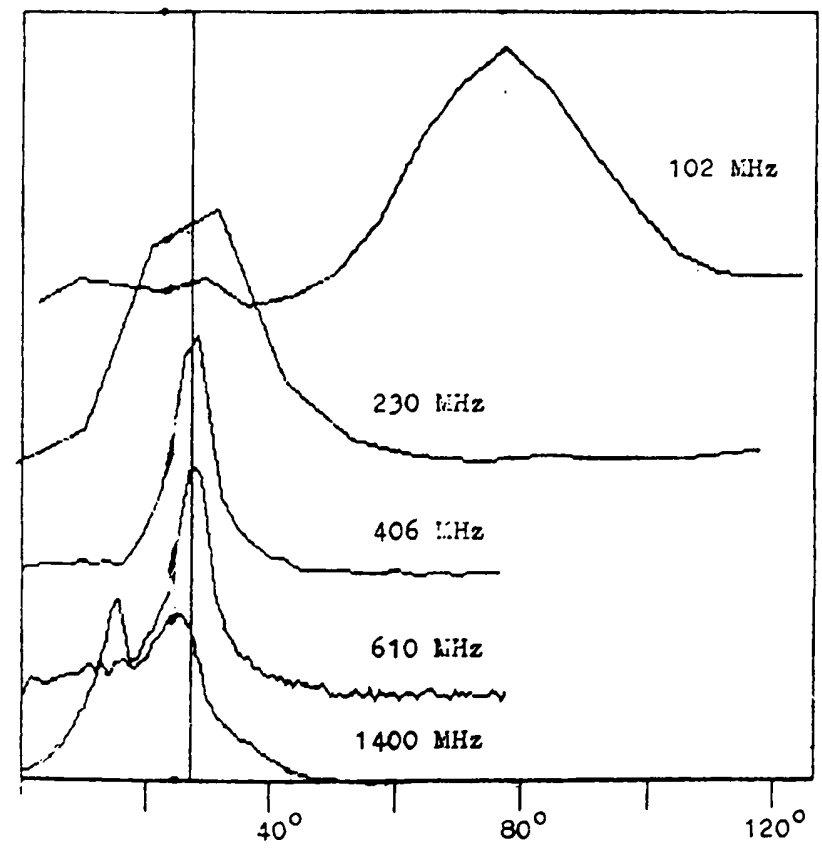

Figure 1a The integrated profiles of PSR 0355+54 aligned with $D M=57.079 \mathrm{pc} \mathrm{cm}^{-3}$.

\section{Observations}

In figure 1a the pulse profiles for PSR $0355+54$ are aligned at the observing frequencies of 610,408 and $230 \mathrm{MHz}$ by choosing the best fitting value of dispersion measure, $D M=57.079 \mathrm{pc} \mathrm{cm}^{-3}$. The profile at $102 \mathrm{MHz}$ then appears delayed unexpectedly by up to 50 degrees. Even if this pulse profile is interpreted as an outer component of a double, which is suggested by the wider profile at $230 \mathrm{MHz}$, there must be a substantial delay at the lowest frequency. In figure $1 \mathrm{~b}$ the profile for PSR 2021+21 also shows a delay at $102 \mathrm{MHz}$ after a similar fitting process. At this stage we should emphasize that our results are critically dependent on the accuracy of timing measurements using a variety of techniques and extending over several observing periods. Assuming

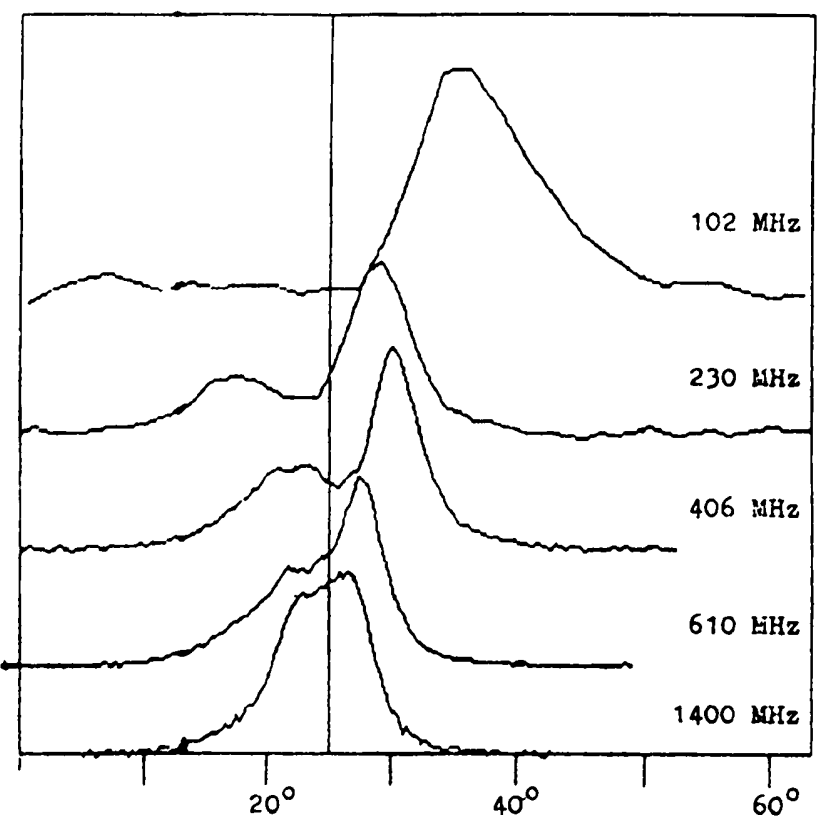

Figure 1b The integrated profiles of PSR 2021+51 aligned with $D M=22.54 \mathrm{pc} \mathrm{cm}^{-3}$. 


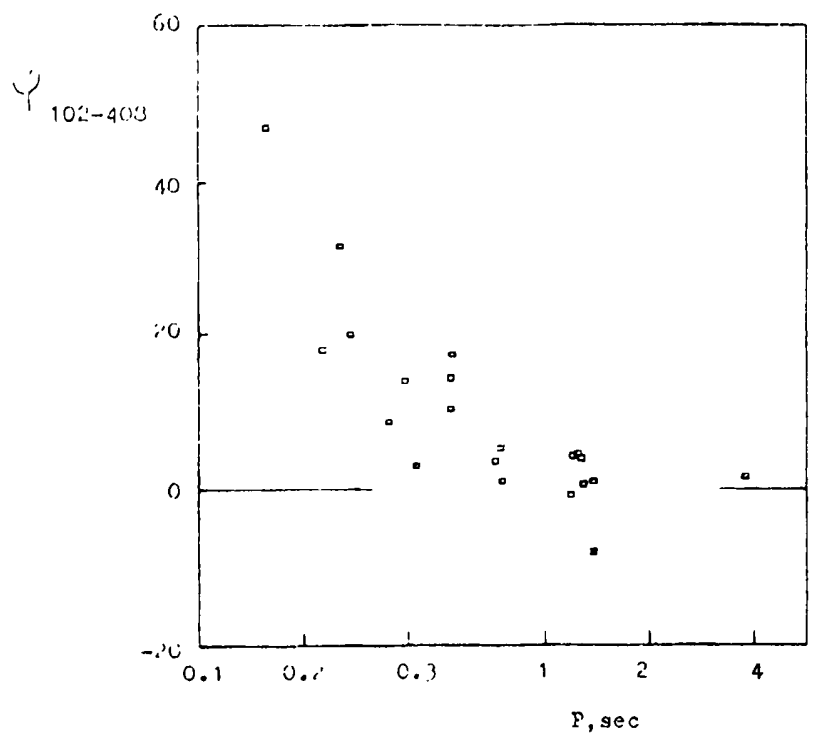

Figure 2 The delay of profiles at $102 \mathrm{MHz}$ in rotation phase as a function of period for 21 pulsars.

that the analysis is correct, we find that these delays occur most commonly in pulsars with short periods. In figure 2 we plot the delays in rotation phase as a function of period for 25 pulsars, showing that the delay rises rapidly for periods below about $0.5 \mathrm{~s}$. The delays which we measure with this technique in some cases do not conform to a simple pattern. An example is provided by PSR 2016+28, shown in figure 3. The profiles for this pulsar are aligned in the same way for frequencies of 610,408 and $320 \mathrm{MHz}$, where our best fitting value of $D M=$ $14.180 \mathrm{pc} \mathrm{cm}^{-3}$ is the same as determined by Hankins and Rickett (1986). But the alignment between

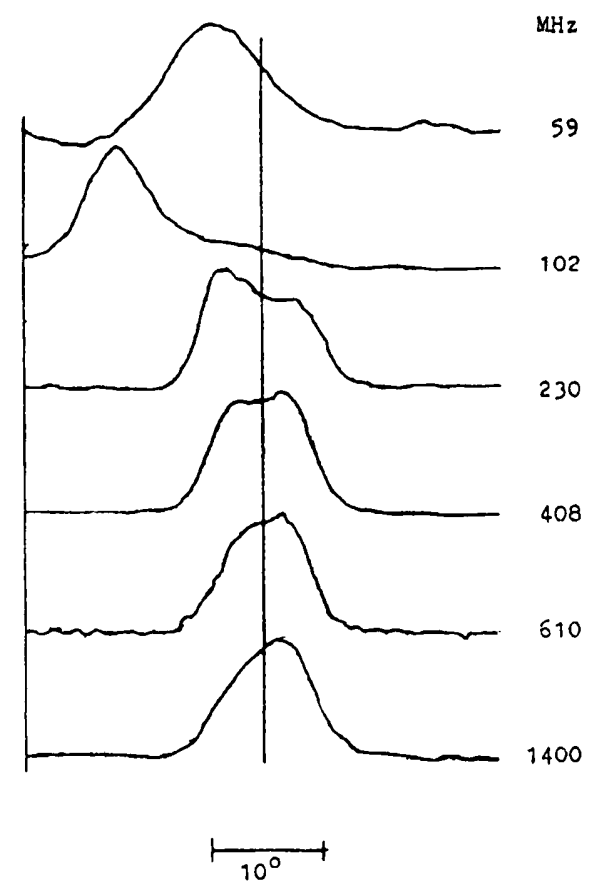

Figure 3 The integrated profiles of PSR 2016+28 aligned with $D M=14.180 \mathrm{pc} \mathrm{cm}^{-3}$.

$102 \mathrm{MHz}$ and higher frequency profiles requires the lower value of $D M=14.14 \mathrm{pc} \mathrm{cm}^{-3}$, which corresponds to a large delay of the profile at $102 \mathrm{MHz}$. If these delays are due to magnetic field twisting, these measurements indicate that the radio emission originates further out in the magnetosphere than the heights estimated from the angular width of the emission cone. Field twisting large enough to account for the observed delays would only be expected for an emission origin at a radial distance of around ten percent of the velocity of light cylinder radius. 